\title{
A computational-based update on microRNAs and their targets in barley (Hordeum vulgare L.)
}

\author{
Moreno Colaiacovo*, Annalisa Subacchi, Paolo Bagnaresi, Antonella Lamontanara, Luigi Cattivelli, Primetta Faccioli
}

\begin{abstract}
Background: Many plant species have been investigated in the last years for the identification and characterization of the corresponding miRNAs, nevertheless extensive studies are not yet available on barley (at the time of this writing). To extend and to update information on miRNAs and their targets in barley and to identify candidate polymorphisms at miRNA target sites, the features of previously known plant miRNAs have been used to systematically search for barley miRNA homologues and targets in the publicly available ESTs database. Matching sequences have then been related to Unigene clusters on which most of this study was based.

Results: One hundred-fifty-six microRNA mature sequences belonging to 50 miRNA families have been found to significantly match at least one EST sequence in barley. As expected on the basis of phylogenetic relations, miRNAs putatively orthologous to those of Triticum are significantly over-represented inside the set of identified barley microRNA mature sequences. Many previously known and several putatively new miRNA/target pairs have been identified. When the predicted microRNA targets were grouped into functional categories, biological processes previously known to be regulated by miRNAs, such as development and response to biotic and abiotic stress, have been highlighted and most of the target molecular functions were related to transcription regulation. Candidate microRNA coding genes have been reported and genetic variation (SNPs/indels) both in functional regions of putative miRNAs (mature sequence) and at miRNA target sites has been found.

Conclusions: This study has provided an update of the information on barley miRNAs and their targets representing a foundation for future studies. Many of previously known plant microRNAs have homologues in barley with expected important roles during development, nutrient deprivation, biotic and abiotic stress response and other important physiological processes. Putative polymorphisms at miRNA target sites have been identified and they can represent an interesting source for the identification of functional genetic variability.
\end{abstract}

\section{Background}

MicroRNAs (miRNAs) are a class of non-coding small RNAs with fundamental roles in key plant biological processes such as development, signal transduction and environmental stress response [1]. miRNAs act on gene regulation at post-transcriptional level, a phenomenon known in plants as PTGS (Post Transcriptional Gene Silencing), through sequence-based interaction with target mRNAs.

Many plant species have been investigated during recent years for miRNAs identification and characterization. The current information available on barley refers to two papers $[2,3]$. In particular, the paper of Dryanova

\footnotetext{
* Correspondence: moreno.colaiacovo@entecra.it
CRA-Genomics Research Centre, via S.Protaso 302, I-29107 Fiorenzuola d'Arda

* Correspondence: moreno.colaiacovo@entecra.it
CRA-Genomics Research Centre, via S.Protaso 302, I-29107 Fiorenzuola d'Arda $(\mathrm{PC})$, Italy
}

(c) 2010 Colaiacovo et al; licensee BioMed Central Ltd. This is an Open Access article distributed under the terms of the Creative Commons Attribution License (http://creativecommons.org/licenses/by/2.0), which permits unrestricted use, distribution, and reproduction in any medium, provided the original work is properly cited.

et al. reports detailed information on both targets and miRNA coding sequences from Hordeum vulgare and for other members of Triticeae tribe, to which barley belongs [2]. However, extensive studies describing the organization of miRNA families, specifically in barley, are not yet available (at the time of this writing) and no miRNAs have been deposited in the publicly available miRNA database (miRBase, http://www.mirbase.org), this despite the economic importance of barley and its role as model species for Triticeae [4].

The conservation of miRNA sequences across species provides a powerful tool for the identification of novel miRNA genes based on homology with miRNAs previously described in other species. Search based on evolutionary conservation has allowed the identification of miRNA families in many plant species, including those 
where the complete genome sequence is not available, as it is currently the case of barley. Without genome sequence information a powerful alternative data source comes from ESTs (Expressed Sequence Tags): currently 501,616 ESTs are available in barley http://www.ncbi.nlm.nih.gov/ dbEST/dbEST_summary.html [5].

The identification of target genes is a fundamental step for the determination of the biological function of microRNAs, besides being an indirect evidence for their existence. Evolutionary conserved targets have proven very helpful to test the effectiveness of miRNA target detection. The perfect or near perfect complementarity between a miRNA and its target mRNA, that is a peculiar feature of plant miRNAs, gives a powerful tool for the identification of target genes through BLAST analysis of miRNA mature sequences vs EST/genomic sequences. A large part of the "in silico" predicted targets have then been confirmed as bona fide targets by experimental approaches including Northern, 5'-RACE and, more recently, degradome analysis via NGS (Next Generation Sequencing) [6,7].

The correct binding of miRNA to its cognate mRNA is critical for regulating the mRNA level and protein expression. This binding can be affected by singlenucleotide polymorphisms or indels in the miRNA target site leading to the suppression of existing binding sites or the generation of illegitimate ones. Therefore, small polymorphisms in miRNA targets can have a relevant effect on gene and protein expression and represent a type of genetic variability that can influence agronomical traits. As an example, overexpression of miR156b and miR156h in rice results in severe dwarfism, strongly reduced panicle size and delayed heading date [8].

To extend and to update information about miRNAs and their targets in barley and to identify candidate polymorphisms at microRNA target sites, barley EST sequences have been screened and related to Unigene clusters. UniGene is an experimental system for partitioning transcript sequences into a non-redundant set of gene-oriented clusters. Thus each UniGene cluster contains sequences that appear to come from the same transcription locus (gene or expressed pseudogene) http://www.ncbi.nlm.nih.gov/UniGene/index.html.

Mining SNPs from ESTs allows the exploitation of genetic variability based on published sequences and the analysis of Unigene clusters can be very helpful for this purpose [9].

\section{Results and Discussion \\ Barley miRNAs}

Since only mature miRNA sequences rather than precursor sequences are conserved among plant species, mature miRNA sequences have been used as queries for
BLAST search against Hordeum vulgare ESTs [10]. One hundred-fifty-six microRNA mature sequences belonging to 50 miRNA families have been found to significantly match at least one EST sequence in barley (the total number of matching ESTs was 855 - as reported in additional files 1 and 2) and could actually be related both to target or miRNA sequences, even if the probability is lower for the latter. Indeed the estimated frequency of pri-miRNAs in T. aestivum EST collection is as low as $0.003 \%$ [2].

The results illustrated above have been compared with those reported by Dryanova et al. where miRNAs and their targets have been searched in the Triticeae tribe [2]. Among the 33 miRNA families identified by Dryanova et al. in at least one species of the Triticeae tribe, 22 families were found in barley and 17 of them overlap with the present findings. Regarding barley, some miRNA families were found in just one of the two papers. Dryanova et al. found evidences for 5 additional miRNA families while the present work has found evidences in barley for miR390 and miR396 previously reported only in $T$. aestivum, and for additional 31 families not found by Dryanova et al. in anyone of the investigated species (i.e. miR442, miR529). The reasons for these discrepancies can be ascribed to the different miRBase release used (miRBase Release 8.0 for Dryanova et al., 2008 and miRBase Release 13.0 in the present work) and partially to differences in the BLAST settings adopted. Monocot-specific miRNAs (i.e. miR444) have also been found in both works [11].

Statistical analysis was employed to identify over and under-represented plant species from which the corresponding barley miRNA comes from. As reported in table 1 and 2, barley miRNA sequences putative orthologous to those of Triticum are significantly overrepresented in our data also when very stringent $\mathrm{p}$ value, e.g. 0.001, was used. Hordeum and Triticum genera are both members of the Poaceae family, Pooideae subfamily, Triticeae tribe. $H$. vulgare is often used as a model species for Triticeae, thanks to its diploid genome that could facilitate genome-wide searches of miRNAs.

Zea mays is also closely related to barley being part of monocot group and Poaceae family. Oryza sativa although is part of Poaceae family is under-represented, when a low stringent p-value $(0.05)$ was used.

Some ESTs have matched to more than one miRNAs belonging either to the same family or to different families (additional file 3). The first case can be due to the high level of similarity among mature sequences from different members of the same family, while ESTs matching to different miRNA families could represent examples of multi-microRNA based control.

Transcripts targeted by more than one miRNA have also been found also in other plant species such as rice 
Table 1 Statistical analysis for the identification of over and under-represented plant species

\begin{tabular}{|c|c|c|c|c|c|}
\hline & \multicolumn{2}{|l|}{ Initial dataset } & \multicolumn{3}{|l|}{ H.vulgare } \\
\hline & $\mathrm{n}^{\circ}$ of mature sequences (redundant set) & $\%$ & $\begin{array}{l}\mathrm{n}^{\circ} \text { of mature sequences (redundant set) } \\
\text { matching at least one barley EST }\end{array}$ & $\%$ & p-value \\
\hline Arabidopsis thaliana & 207 & 10.7 & 43 & 8.7 & 0.019 \\
\hline Oryza sativa & 415 & 21.5 & 102 & 20.5 & 0.038 \\
\hline Glycine max & 79 & 4.1 & 15 & 3.0 & 0.046 \\
\hline Pinus taeda & 38 & 2.0 & 6 & 1.2 & 0.068 \\
\hline Triticum aestivum & 32 & 1.7 & 20 & 4.0 & $2.0 \times 10^{-4}$ \\
\hline Physcomitrella patens & 281 & 14.6 & 39 & 7.8 & $1.7 \times 10^{-6}$ \\
\hline Populus trichocarpa & 237 & 12.3 & 71 & 14.3 & 0.021 \\
\hline Chlamydomonas reinhardtii & 84 & 4.4 & 2 & 0.4 & $6.3 \times 10^{-8}$ \\
\hline Selaginella moellendorffii & 64 & 3.3 & 12 & 2.4 & 0.058 \\
\hline Vitis vinifera & 140 & 7.3 & 47 & 9.5 & 0.012 \\
\hline Brassica napus & 44 & 2.3 & 19 & 3.8 & 0.010 \\
\hline Gossypium hirsutum & 13 & 0.7 & 4 & 0.8 & 0.185 \\
\hline Medicago truncatula & 46 & 2.4 & 8 & 1.6 & 0.068 \\
\hline Solanum lycopersicum & 30 & 1.6 & 11 & 2.2 & 0.065 \\
\hline Sorghum bicolor & 72 & 3.7 & 27 & 5.4 & 0.014 \\
\hline Zea mays & 98 & 5.1 & 48 & 9.7 & $1.1 \times 10^{-5}$ \\
\hline Brassica oleracea & 7 & 0.4 & 2 & 0.4 & 0.268 \\
\hline Brassica rapa & 19 & 1.0 & 8 & 1.6 & 0.061 \\
\hline Saccharum officinarum & 16 & 0.8 & 11 & 2.2 & $2.3 \times 10^{-3}$ \\
\hline Gossypium herbecium & 1 & 0.1 & 0 & 0.0 & 0.773 \\
\hline Carica papaya & 1 & 0.1 & 0 & 0.0 & 0.773 \\
\hline Vigna unguiculata & 1 & 0.1 & 0 & 0.0 & 0.773 \\
\hline Lotus japonicus & 2 & 0.1 & 0 & 0.0 & 0.597 \\
\hline Gossypium rammindii & 2 & 0.1 & 2 & 0.4 & 0.079 \\
\hline Total & 1929 & & 497 & & \\
\hline
\end{tabular}

For each species, the table shows the number of mature sequences from the redundant set of 1929 sequences stored in the miRBase and number of mature sequences matching at least one barley EST. It also shows the p-value calculated with a binomial distribution.

[12]. These findings are common in animals where many different miRNAs recognize the same target mRNA, usually at the 3'UTR [13].

To identify and annotate potential microRNA-regulated genes in barley, the 855 matching ESTs were related to Unigene clusters. Clusters annotated as protein-coding sequences were then selected for subsequent analysis and listed in tables 3 and 4 . A total of 121 different Unigene clusters putatively representing the targets for 37 miRNA families has been found. Similar results (e.g. on average more than 1 putative target/ miRNA family) were reported by Zhang et al. in maize (115 target for 26 miRNA families) [14]. Sometimes different targets for a specific miRNA are members of the same gene family (e.g. miR156-SBP family), while in other cases there is no evident relationship among the putative targets of a given miRNA (e.g. miR1121). Previous studies report six targets or fewer for most Arabidopsis miRNAs, a number significantly lower than in animals, for example, in Drosophila each miRNA has on average over 50 predicted targets $[13,15]$.

Although several of the candidate miRNA/target pairs here identified have the same functional annotation reported in previously studied species (table 3 ) and specifically in barley some putative novel microRNA/target pairs have been discovered (table 4) [2]. Actually, some of these novel targets were reported by literature as regulated by a different microRNA. Most of the novel miRNA/target pairs refer to miRNAs recently discovered and thus probably less studied (i.e. miR1120, miR1122, miR1134). The Argonaute-like protein found as a novel target for miR408 in H.vulgare by Dryanova et al. has been confirmed also in the present work.

Transcription factor families comprise most of the highly conserved miRNA targets (see table 3 ) such as SBP family for miRNA 156, AP2 family for miR172, GRAS family for miR171, myb family for miR159, GRF family for miR396 and ARF family for miR160. These 
Table 2 Over and under-represented plant species within barley miRNAs identified with respect to the stringency chosen for the p-value

\begin{tabular}{lll}
\hline Threshold & Over-represented plant species & Under-represented plant species \\
\hline $\mathrm{p}$-value $\leq 0.05$ & Triticum aestivum & Arabidopsis thaliana \\
& Populus trichocarpa & Oryza sativa \\
& Vitis vinifera & Glycine max \\
& Brassica napus & Physcomitrella patens \\
& Sorghum bicolor & Chlamydomonas reinhardtii \\
& Zea mays & \\
& Saccharum officinarum & \\
\hline $\mathrm{p}$-value $\leq 0.01$ & Triticum aestivum & Physcomitrella patens \\
& Zea mays & Chlamydomonas reinhardtii \\
\hline $\mathrm{p}$-value $\leq 0.005$ & Saccharum officinarum & \\
\hline $\mathrm{p}$-value $\leq 0.001$ & Triticum aestivum & Physcomitrella patens \\
& Zea mays & Chlamydomonas reinhardtii \\
\hline & Saccharum officinarum & \\
\hline
\end{tabular}

results confirmed what previously observed in Triticeae and in other species [2]. In rice about $70 \%$ of conserved miRNA targets are transcription factors, while in wheat one-third of the predicted targets was found to encode for transcription factors $[11,12]$. Conserved miRNAs also target genes involved in their own biogenesis and function: as an example miR168 targets AGO1 which is part of the RISC complex responsible for the miRNAmediated mRNA cleavage [15]. miRNA regulate gene expression also by targeting enzymes of the ubiquitination pathway (ubiquitin conjugating enzyme E2 and TIR1/ubiquitin ligase): barley miR393, miR399, miR1128, miR1133, miR1135 can be considered putative regulators of gene expression at protein level.

The number of target genes identified as different Unigene clusters (tables 3-4) is very different among the miRNA families. In rice Zhou et al. have found a high number of targets for miR156 and miR396 and a low number for miR162, miR167, miR395, miR398 and miR399 [12]. This finding could indicate that the former miRNAs are nodes in gene regulation networks, while the latter could act on specialized pathways.

The predicted targets have been grouped into functional categories and reported in figures 1 and 2 where the target annotations based on GO terms are shown. Biological processes known to be regulated by miRNAs, such as development and response to biotic and abiotic stress, have been highlighted both in known (figure 1a) and in novel targets (figure 2a). Moreover, most of the molecular functions are related to transcriptional regulation and DNA/nucleotide binding in both groups (figures $1 \mathrm{~b}-2 \mathrm{~b}$ ). These findings suggest that the predicted target genes can be considered a reliable dataset to be used in subsequent analysis.

For some Unigene clusters the annotation was related to transcribed genes rather than protein coding sequences. These Unigenes could represent miRNAcoding genes as shown by other authors $[16,17]$. Table 5 reports the Unigene clusters candidate to encode miRNA coding genes on the basis of the precursor sequence secondary structure (MFEI $>0.85$, see Materials and Methods) and of the presence of the miRNA* (miRNA passenger sequence). It cannot be excluded that the clusters unable to fold with a miRNA-like structure (e.g. Hv.8579, Hv.11623) are false negatives for several reasons, such as truncated precursor sequences in EST database. Putative microRNA sequences have also been BLASTed against previously known precursors available from mirBASE: the analysis found similarities with 6 different miRNA families. The secondary structures of the putative microRNA precursors are reported in the additional file 4. Linking together sequences containing miRNA precursors from Dryanova et al. and from the present work, information on several microRNA putative secondary structures, belonging to 10 miRNA families are now available [2]. The mature miRNAs predicted from these data are 18 to 24 nt long, with a higher frequency for 20 and $21 \mathrm{nt}$.

\section{Genetic variation at miRNA target sites}

A single nucleotide change in the sequence of a target site can affect miRNA regulation: as a consequence naturally occurring SNPs in target sites are candidates for relevant functional variations. Nair et al. established 
Table 3 miRNA target genes identified in barley and confirmed by previous studies

\begin{tabular}{|c|c|c|c|c|}
\hline $\begin{array}{l}\text { miRNA } \\
\text { family }\end{array}$ & $\begin{array}{c}\text { miRNA } \\
\text { name }\end{array}$ & Unigene & Unigene annotation & $\begin{array}{c}\text { Literature reported target for this miRNA (citation } \\
\text { number in brackets) }\end{array}$ \\
\hline \multirow[t]{6}{*}{156} & miR156 & Hv.29207 & protein coding (SBP domain) & Arabidopsis thaliana [24] \\
\hline & miR156 & Hv.5875 & protein coding (SBP domain) & Oryza sativa [8] \\
\hline & miR156 & Hv.28351 & protein coding (SBP domain) & Hordeum vulgare [2] \\
\hline & miR156 & Hv.21387 & $\begin{array}{l}\text { SPL2 (SQUAMOSA PROMOTER BINDING } \\
\text { PROTEIN-LIKE 2) }\end{array}$ & Triticum aestivum [2] \\
\hline & miR156 & Hv.28414 & $\begin{array}{l}\text { SPL5 (SQUAMOSA PROMOTER BINDING } \\
\text { PROTEIN-LIKE 5) }\end{array}$ & \\
\hline & & & & Arabidopsis thaliana [24] \\
\hline \multirow[t]{4}{*}{159} & miR159 & Hv.12 & MYB family transcription factor & Oryza sativa [39] \\
\hline & & & & Hordeum vulgare [2] \\
\hline & & & & Triticum aestivum [2] \\
\hline & & & & Arabidopsis thaliana [24] \\
\hline \multirow[t]{3}{*}{160} & miR160 & Hv.5089 & ARF16 (AUXIN RESPONSE FACTOR 16) & Oryza sativa [39] \\
\hline & & & & Hordeum vulgare [2] \\
\hline & & & & Triticum aestivum [2] \\
\hline \multirow[t]{4}{*}{164} & miR164 & Hv.877 & NAC domain containing protein & Arabidopsis thaliana [24] \\
\hline & miR164 & Hv.28795 & NAC domain containing protein & Zea mays [14] \\
\hline & miR164 & Hv. 25370 & NAM superfamily & Hordeum vulgare [2] \\
\hline & miR164 & Hv.21779 & NAC domain containing protein & Triticum aestivum [2] \\
\hline \multirow[t]{3}{*}{168} & miR168 & Hv.26206 & AGO1 (ARGONAUTE 1) & Arabidopsis thaliana [24] \\
\hline & miR168 & Hv.19452 & AGO1 (ARGONAUTE 1) & Hordeum vulgare [2] \\
\hline & & & & Triticum aestivum [2] \\
\hline \multirow[t]{4}{*}{169} & miR169 & Hv.13681 & $\begin{array}{l}\text { CCAAT-binding transcription factor (CBF-B/NF-YA) } \\
\text { family protein }\end{array}$ & Aquilegia coerulea [40] \\
\hline & $\operatorname{miR169}$ & Hv.406 & $\begin{array}{l}\text { CCAAT-binding transcription factor (CBF-B/NF-YA) } \\
\text { family protein }\end{array}$ & Hordeum vulgare [2] \\
\hline & miR169 & Hv.9532 & $\begin{array}{l}\text { CCAAT-binding transcription factor (CBF-B/NF-YA) } \\
\text { family protein }\end{array}$ & Triticum aestivum [2] \\
\hline & & & & Arabidopsis thaliana [24] \\
\hline \multirow[t]{4}{*}{171} & miR171 & Hv.9855 & GRAS family transcription factor & Brachypodium distachyon [41] \\
\hline & & & & Hordeum vulgare [2] \\
\hline & & & & Triticum aestivum [2] \\
\hline & & & & Arabidopsis [42] \\
\hline \multirow[t]{2}{*}{172} & miR172 & Hv. 6575 & RAP2.7/TOE1 (TARGET OF EAT1 1), AP2 superfamily & Hordeum vulgare [2] \\
\hline & & & & Triticum aestivum [2] \\
\hline \multirow[t]{4}{*}{393} & miR393 & Hv.29376 & $\begin{array}{c}\text { AFB2 (AUXIN SIGNALING F-BOX 2), auxin binding/ } \\
\text { ubiquitin-protein ligase }\end{array}$ & Aquilegia coerulea [40] \\
\hline & miR393 & Hv.2498 & $\begin{array}{c}\text { TIR1 (TRANSPORT INHIBITOR RESPONSE 1), ubiquitin- } \\
\text { protein ligase }\end{array}$ & Hordeum vulgare [2] \\
\hline & & & & Triticum aestivum [2] \\
\hline & & & & Aquilegia coerulea [40] \\
\hline \multirow[t]{3}{*}{394} & miR394 & Hv.8877 & F-box family protein & Hordeum vulgare [2] \\
\hline & & & & Triticum aestivum [2] \\
\hline & & & & Aquilegia coerulea [40] \\
\hline \multirow[t]{2}{*}{395} & miR395 & Hv.12870 & ATPS1 & Hordeum vulgare [2] \\
\hline & & & & Triticum aestivum [2] \\
\hline \multirow[t]{4}{*}{396} & miR396 & Hv.28722 & WRC, QLQ & \\
\hline & miR396 & Hv.22031 & growth-regulating factor & Arabidopsis thaliana [39] \\
\hline & miR396 & Hv.19321 & WRC, QLQ & Oryza sativa [39] \\
\hline & miR396 & Hv.9742 & WRC, QLQ & Triticum aestivum [2] \\
\hline
\end{tabular}


Table 3 miRNA target genes identified in barley and confirmed by previous studies (Continued)

\begin{tabular}{|c|c|c|c|c|}
\hline 399 & miR399 & Hv. 5443 & $\begin{array}{l}\text { ATUBC24/PHO2/UBC24 (PHOSPHATE 2), ubiquitin- } \\
\text { protein ligase }\end{array}$ & $\begin{array}{c}\text { Arabidopsis thaliana [39] } \\
\text { Oryza sativa [39] }\end{array}$ \\
\hline \multirow[t]{6}{*}{408} & miR408 & Hv.10831 & $\begin{array}{l}\text { ARPN (PLANTACYANIN), copper ion binding (Cu-bind- } \\
\text { like superfamily) }\end{array}$ & Medicago truncatula [43] \\
\hline & & & & Populus trichocarpa [44] \\
\hline & miR408 & Hv.24052 & $\begin{array}{l}\text { Plastocyanin-like domain-containing protein (Cu-bind- } \\
\text { like superfamily) }\end{array}$ & Oryza sativa [45] \\
\hline & & & & Hordeum vulgare [2] \\
\hline & & & & Triticum aestivum [2] \\
\hline & miR408 & Hv.20945 & ARGONAUTE like superfamily & Hordeum vulgare [2] \\
\hline \multirow[t]{3}{*}{529} & miR529 & Hv.29207 & protein coding (SBP domain) & Aquilegia coerulea [40] \\
\hline & miR529 & Hv.28351 & protein coding (SBP domain) & Zea Mays [46] \\
\hline & & & & Arabidopsis thaliana [47] \\
\hline 827 & miR827 & HV. 10218 & SPX superfamily, MFS superfamily & Oryza sativa $[48]$ \\
\hline
\end{tabular}

a perfect association between a SNP at the miR172 targeting site and cleistogamy in barley [18]. Overall few papers have been published to date describing variations among plant genotypes at miRNAs and their target sites, while plenty of information is available for humans [19-23]. Genome-wide studies in humans have shown that the levels of polymorphism at miRNA and miRNA target sites are lower than at coding or neutral regions, however beneficial miRNA-target site polymorphisms also exist [19].

In this study, publicly available SNP data have been analyzed in context with miRNAs and their target sites. EST-derived SNPs can provide a rich source of biologically useful genetic variation due to the redundancy of gene sequence, the diversity of genotypes present in the databases and the fact that each putative polymorphism is associated with an expressed gene. Variations both in functional regions of putative miRNAs (mature sequence) and at miRNA target sites have been found. Previous works in human have highlighted a relatively low level of variation in functional microRNA regions and an appreciable level of variation at target sites [21].

Hv.5064, the candidate for miR1137 coding sequence, has been tested for modifications of pre-miRNA structure due to a base substitution in position $13(\mathbf{C} / \mathbf{G}$, table 6, figure 3). To evaluate the possible impact of this SNP on pre-miRNA secondary structure, Gibbs free energy $(\Delta G)$ and MFEI from each version of premiRNA were calculated using mfold program. Data in figure 3 show the structural variation obtained when moving from "C variant" to " $G$ variant" with a higher MFEI for the second one and thus a greater stability of the molecule (miRNA-miRNA* pairing enhanced in the $\mathrm{G}$ variant). Difference in $\Delta \mathrm{G}$ moving from $\mathrm{C}$ to $\mathrm{G}$ and vice versa were calculated according to Ehrenreich and
Purugganan [19]. $\Delta \Delta G$ was +1.3 for the former change and -1.3 for the latter suggesting that some SNPs can stabilize/destabilize pre-miRNA structure. No target gene has been reported in literature for miR1137.

In plants most of the miRNA-based regulation relies on the cleavage of target mRNAs that normally occurs at the tenth nucleotide of the complementary region and numerous studies on miRNA-target interaction have highlighted the importance of positions 2 to 12 , more frequently 10 and 11 [24]. Although most of the putative polymorphisms highlighted in this work are outside those critical positions, several examples of putative functionally relevant polymorphisms have been detected. Table 6 reports the putative polymorphisms detected after comparison among EST sequences inside Unigene clusters, without any selection against false positives. Some of these nucleotide variation could be due to sequencing errors or related to very similar genes belonging to a specific family, nevertheless when the SNPs/indels rely on two or more copies of independent sequences it can be considered a good candidate for a true positive polymorphic target site [25]. For example, a polymorphism in miRNA 408 target site detected by AutoSNP in contig 2094 (coding for a plastocyanin) is based on sequences from two different cultivars reporting the same allelic variant as part of a haplotype where a SSR (Simple Sequence Repeat) polymorphism is located upstream the target sequence (figure 4). Some polymorphisms also showed an evolutionary conserved position, the nucleotide variation identified in Hv.2498 (targeted by miR393) has also been found in the orthologous gene of Arabidopsis in the same position by Ehrenreich and Purugganan [19].

The Squamosa-promoter Binding Protein (SBP) is a known target family for miR156. Many plant transcription 
Table 4 Novel miRNA target genes identified

\begin{tabular}{|c|c|c|c|c|}
\hline $\begin{array}{l}\text { miRNA } \\
\text { family }\end{array}$ & $\begin{array}{l}\text { miRNA } \\
\text { name }\end{array}$ & Unigene & Unigene annotation & Functional annotation \\
\hline 390 & miR390 & Hv.15993 & protease inhibitor, seed storage, lipid transfer protein (LTP) family protein & lipid transport \\
\hline \multirow[t]{4}{*}{441} & miR1126 & Hv.10635 & beta-adaptin & protein transport \\
\hline & $\operatorname{miR} 1126$ & Hv.25101 & ankyrin protein kinase, serine/threonine protein kinase & regulation in signal transduction \\
\hline & $\operatorname{miR} 1126$ & Hv.18172 & protein coding & unknown function \\
\hline & miR1126 & Hv.5267 & SRT2, DNA binding & vernalization, auxin signalling \\
\hline \multirow[t]{16}{*}{818} & $\begin{array}{l}\text { miR818 } \\
+1436\end{array}$ & Hv.11323 & protein coding & unknown function \\
\hline & $\begin{array}{l}\operatorname{miR} 818 \\
+1436\end{array}$ & Hv.9623 & NLI interacting factor (NIF) family protein & phosphatase activity \\
\hline & miR1436 & Hv.8609 & Coproporphyrinogen III oxidase & chlorophyll biosynthesis \\
\hline & miR1436 & Hv.16854 & P-loop NTPase superfamily & unknown function \\
\hline & $\operatorname{miR} 1436$ & Hv.8351 & protein coding & unknown function \\
\hline & miR1436 & Hv.28025 & protein coding & unknown function \\
\hline & miR1436 & Hv.27779 & Vps51 superfamily & vescicular transport \\
\hline & miR1436 & Hv.19811 & ILL3 (IAA-amino acid hydrolase ILR1-like 3), metallopeptidase & stress and hormone response \\
\hline & miR1436 & Hv.18734 & MAP kinase & signal transduction, stress signalling \\
\hline & miR1436 & Hv.15543 & protein coding & unknown function \\
\hline & miR1436 & Hv.12920 & PKc-like superfamily & abiotic stress resistance \\
\hline & miR1436 & Hv.11057 & Integral membrane family protein & endomembrane system \\
\hline & miR1436 & Hv.3476 & protein coding & unknown function \\
\hline & miR1439 & Hv.19109 & PKc-like superfamily & unknown function \\
\hline & miR1439 & Hv.23816 & exo-endo-phos superfamily & unknown function \\
\hline & miR1439 & Hv.11224 & tatD-related deoxyribonuclease family protein & deoxyribonuclease activity \\
\hline 821 & miR821 & Hv.3660 & GDH1 (Glutamate dehydrogenase) & nitrogen metabolism \\
\hline \multirow[t]{3}{*}{1030} & miR1030 & Hv.12064 & $\begin{array}{l}\text { AS1/ATMYB91/ATPHAN/MYB91 (ASYMMETRIC LEAVES 1, MYB DOMAIN } \\
\text { PROTEIN) }\end{array}$ & transcription factor \\
\hline & miR1030 & Hv.7960 & protein coding & unknown function \\
\hline & miR1030 & Hv.14867 & RNA recognition motif (RRM)-containing protein & $\begin{array}{c}\text { post-transcriptional gene expression } \\
\text { processes }\end{array}$ \\
\hline \multirow[t]{6}{*}{1119} & miR1119 & Hv.29225 & protein coding & unknown function \\
\hline & $\operatorname{miR} 1119$ & Hv.29210 & protein coding & unknown function \\
\hline & $\operatorname{miR} 1119$ & Hv.27666 & protein coding & unknown function \\
\hline & miR1119 & Hv.23883 & ADF2 (ACTIN DEPOLYMERIZING FACTOR 2), actin binding & $\begin{array}{l}\text { actin turnover, stress response, plant } \\
\text { defense signalling pathway }\end{array}$ \\
\hline & $\operatorname{miR} 1119$ & Hv.23689 & RRM superfamily, RNA binding & $\begin{array}{l}\text { involved in post-transcriptional gene } \\
\text { expression processes }\end{array}$ \\
\hline & miR1119 & Hv.23343 & molybdenum cofactor sulfurase family protein, superfamily & stress response \\
\hline \multirow[t]{10}{*}{1120} & $\operatorname{miR} 1120$ & Hv.21827 & protein coding & unknown function \\
\hline & miR1121 & Hv.464 & serine/threonine kinase & response to salt stress \\
\hline & miR1121 & Hv.20180 & Kelch repeat-containing protein & unknown function \\
\hline & miR1121 & Hv.2132 & protein coding & unknown function \\
\hline & $\operatorname{miR} 1121$ & Hv.26959 & POK (POKY POLLEN TUBE) & pollen tube growth \\
\hline & $\operatorname{miR} 1121$ & Hv.20763 & SRG1 (SENESCENCE-RELATED GENE 1), oxidoreductase & $\begin{array}{l}\text { flavonoid biosyntetic processes and } \\
\text { senescence }\end{array}$ \\
\hline & miR1121 & Hv.20600 & serine/threonine protein kinase, PKc-like superfamily & abiotic stress resistance \\
\hline & $\operatorname{miR} 1121$ & Hv.12124 & ATPase family AAA domain-containing protein & unknown function \\
\hline & $\operatorname{miR} 1121$ & Hv.10391 & protein coding & unknown function \\
\hline & miR1121 & Hv.9294 & protein coding & unknown function \\
\hline
\end{tabular}


Table 4 Novel miRNA target genes identified (Continued)

\begin{tabular}{|c|c|c|c|c|}
\hline & miR1121 & Hv.6581 & protein coding & unknown function \\
\hline & $\operatorname{miR} 1121$ & Hv.6532 & ATPase-Plipid, haloacid dehalogenase-like hydrolase family protein & ATPase activity \\
\hline & $\operatorname{miR} 1121$ & Hv.4756 & FAR1 superfamily, MULE transposon domain & light control of development \\
\hline & miR1121 & Hv.3142 & CRS1-YhbY (CRM domain) superfamily & RNA binding/intron splicing \\
\hline \multirow[t]{21}{*}{1122} & miR1122 & Hv.12219 & serine/threonine protein kinase, PKc-like superfamily & abiotic stress resistance \\
\hline & $\begin{array}{l}\operatorname{miR} 1128 \\
+1133\end{array}$ & Hv.23560 & indole-3-glycerol phosphate synthase, TIM-phosphate binding superfamily & aminoacid biosynthesis \\
\hline & $\begin{array}{l}\operatorname{miR} 1128 \\
+1133\end{array}$ & Hv.679 & UBIQUITIN CARRIER PROTEIN, ubiquitin-protein ligase & ubiquitination \\
\hline & $\begin{array}{l}\operatorname{miR} 1128+ \\
1133+1136\end{array}$ & Hv.26146 & AIM1 (ABNORMAL INFLORESCENCE MERISTEM), enoyl-CoA hydratase & auxin metabolism \\
\hline & $\begin{array}{l}\operatorname{miR} 1128 \\
+1135\end{array}$ & Hv.23257 & integral membrane HPP family protein & unknown function \\
\hline & $\begin{array}{l}\operatorname{miR} 1128 \\
+1135\end{array}$ & Hv.21122 & SOS5 (SALT OVERLY SENSITIVE 5) & salt signalling/osmo-stress \\
\hline & $\operatorname{miR} 1128$ & Hv.17314 & protein coding & unknown function \\
\hline & miR1128 & Hv.14876 & ARF-GAP DOMAIN, C2 superfamily & vescicle traffic/development \\
\hline & $\begin{array}{l}\operatorname{miR} 1128 \\
+1133\end{array}$ & Hv.12752 & ATP-dependent peptidase, ATPase, metallopeptidase & peptidase activity \\
\hline & $\begin{array}{l}\operatorname{miR} 1128+ \\
1133+1136\end{array}$ & Hv.6454 & oligopeptide transporter & oligopeptide transporter \\
\hline & $\begin{array}{l}\operatorname{miR} 1128 \\
+1133\end{array}$ & Hv.3596 & Cysteine hydrolases, catalytic/nicotinamidase & response to abscisic acid stimulus \\
\hline & miR1133 & Hv.14592 & pathogenesis related protein-1 & plant defense \\
\hline & miR1133 & Hv.12091 & oxidoreductase, zinc-binding dehydrogenase family protein & stress response \\
\hline & miR1133 & Hv.28954 & HLH superfamily & transcription factor \\
\hline & miR1133 & Hv.28555 & serine/threonine protein kinase, PKc-like superfamily & abiotic stress resistance \\
\hline & miR1133 & Hv.4244 & CTP synthase & CTP synthase activity \\
\hline & miR1135 & Hv. 5272 & Epidermal growth factor receptor-like protein & vacuolar transport \\
\hline & miR1135 & Hv.223 & Limit dextrinase & carbohydrate metabolic process \\
\hline & miR1135 & Hv.18515 & ubiquitin family protein & ubiquitination \\
\hline & miR1135 & Hv.16976 & HEAT repeat-containing protein & unknown function \\
\hline & miR1135 & Hv.16897 & $\begin{array}{c}\text { ATTPS6 (A. thaliana trehalose phosphatase/synthase 6), transferase, } \\
\text { transferring glycosyl groups, trehalose-phosphatase }\end{array}$ & development \\
\hline 1130 & miR1130 & Hv.12920 & PKc-like superfamily & abiotic stress response \\
\hline \multirow[t]{11}{*}{1134} & miR1134 & Hv.29810 & WRKY transcription factor & transcription factor \\
\hline & miR1134 & Hv.29222 & ribulose-1,5-bisphosphate carboxylase/oxygenase large subunit & carbon fixation \\
\hline & miR1134 & Hv.22973 & $\begin{array}{l}\text { octopine synthase binding factor1, ATBZIP53 (BASIC REGION/LEUCINE } \\
\text { ZIPPER MOTIF 53), DNA binding/protein heterodimerization/sequence- } \\
\text { specific DNA binding/transcription factor }\end{array}$ & stress response \\
\hline & miR1134 & Hv.22600 & fumarylacetoacetate hydrolase family protein & tyrosine catabolism \\
\hline & miR1134 & Hv.9579 & L-asparaginase, putative/L-asparagine amidohydrolase, putative & nitrogen metabolism \\
\hline & miR1134 & Hv.239 & AWPM-19-like membrane family protein & freezing tolerance \\
\hline & miR1134 & Hv.26138 & AWPM-19-like membrane family protein & freezing tolerance \\
\hline & miR1134 & Hv.24001 & dehydrin family protein & stress response \\
\hline & miR1134 & Hv.23108 & B3-hordein fragment & seed storage protein \\
\hline & miR1134 & Hv.23080 & ATNUDT17 (A. thaliana Nudix hydrolase homolog 17) & hydrolase activity \\
\hline & miR1134 & Hv.16060 & Sulfotransferase domain & sulfotransferase activity \\
\hline 1438 & miR1438 & Hv.26216 & RAP2.2, AP2 superfamily & transcription factor \\
\hline 1533 & miR1533 & Hv.29041 & aldehyde dehydrogenase & stress response \\
\hline 1846 & miR1846 & Hv.19467 & UDP-GLUCOSYL TRANSFERASE & stress response \\
\hline
\end{tabular}


Table 4 Novel miRNA target genes identified (Continued)

\begin{tabular}{ccccc}
\hline 1848 & miR1848 & Hv.6944 & Pollen_Ole_e_I super family & unknown function \\
\hline 1862 & miR1862 & Hv.26602 & protein coding & unknown function \\
\hline 1867 & miR1867 & Hv.18578 & FLAVODOXIN-LIKE QUINONE REDUCTASE 1 & auxin response gene \\
\hline & miR1867 & Hv.1368 & ATPase, coupled to transmembrane movement of substances & ATPase activity \\
\hline 1871 & miR1871 & Hv.28885 & protein coding & unknown function \\
\hline 2091 & miR2091 & Hv.6058 & FKBP superfamily & $\begin{array}{c}\text { regulation of photosyntetic process/ } \\
\text { stress response/plant hormone } \\
\text { pathways }\end{array}$ \\
\hline 2094 & miR2094 & Hv.699 & RNA binding & RNA binding \\
\hline 2102 & miR2102 & Hv.22799 & RNA binding & stress response \\
\hline
\end{tabular}

factors involved in the regulation of the transition from the vegetative to the reproductive phase belong to this family and it has been shown that overexpressing SBP genes can lead to increased leaf initiation, decreased apical dominance and delayed flowering time [15]. The increase of the activity of some miRNAs (among which miR156) is part of the infection strategy performed by the Turnip mosaic virus in Arabidopsis [26,27]. miR156 performs a critical function in mediating developmental processes and it is also related to the response to biotic stress. The
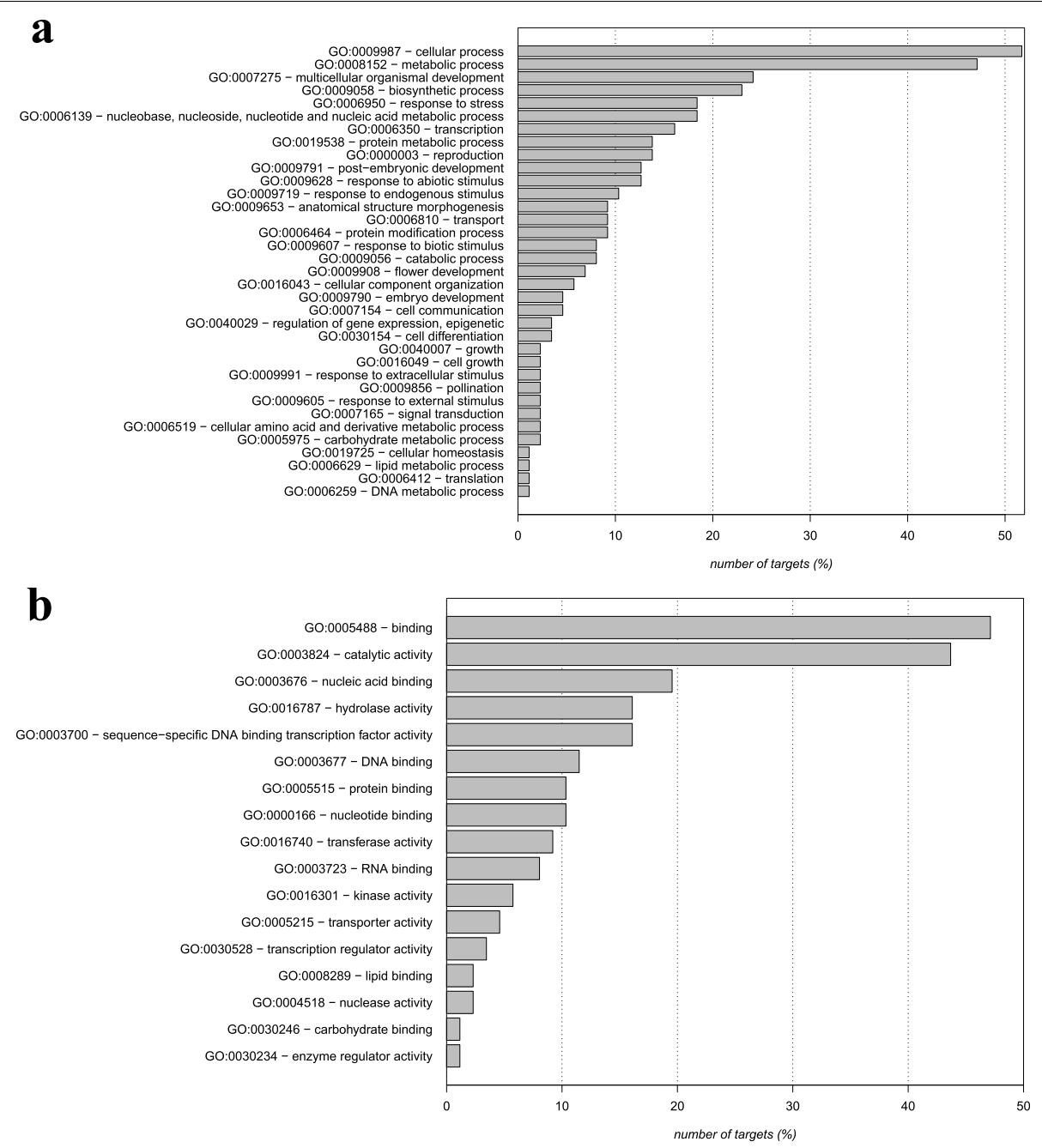

Figure 1 Functional enrichment for the miRNA targets identified. For each GO term it is shown the number of targets annotated with that term with respect to the total number of targets (\%). Figure 1a refers to the biological process, while figure $1 \mathrm{~b}$ refers to the molecular function. 

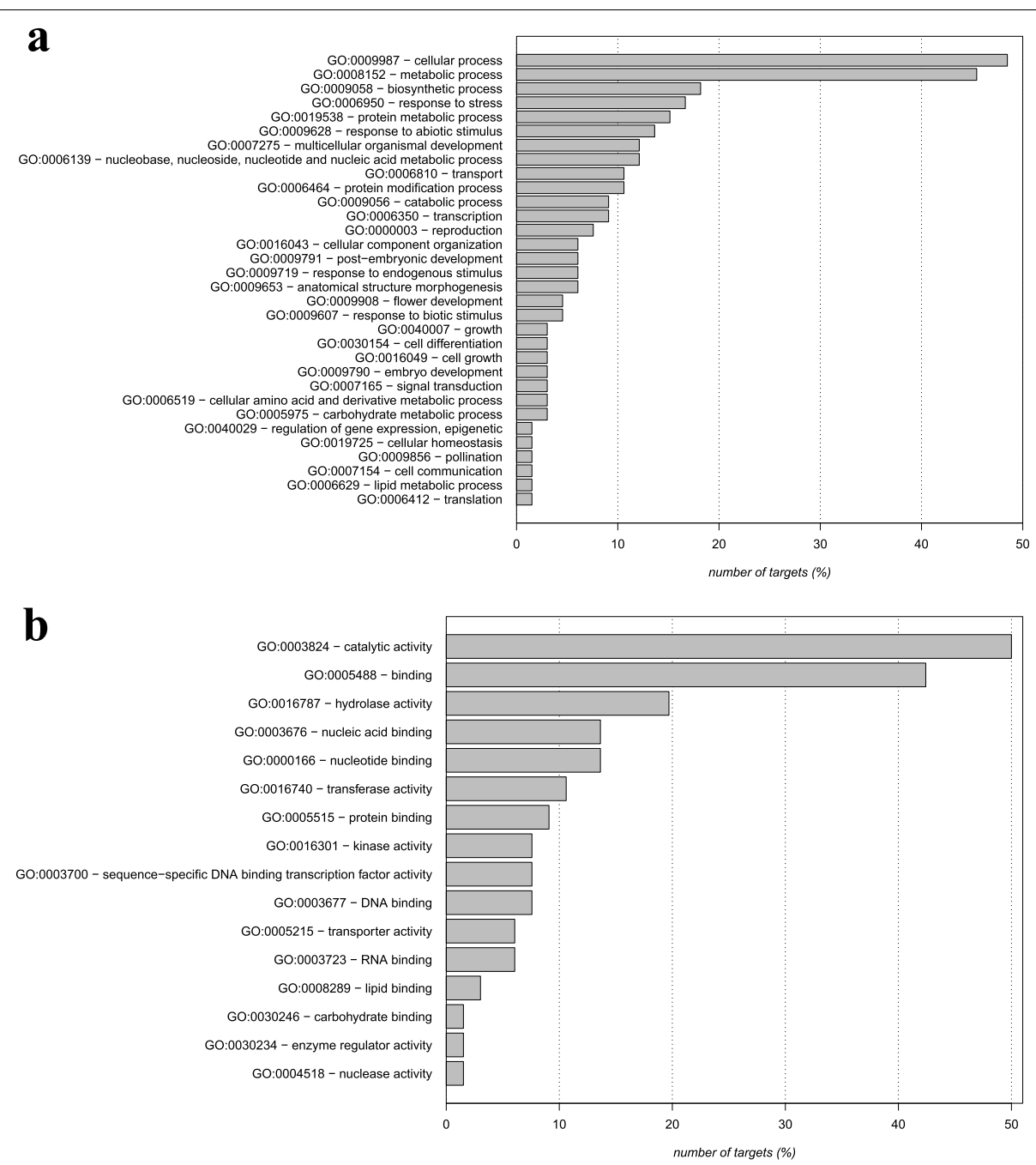

Figure 2 Functional enrichment for the novel miRNA targets identified. For each GO term it is shown the number of targets annotated with that term with respect to the total number of novel targets (\%). Figure $2 a$ refers to the biological process, while figure $2 \mathrm{~b}$ refers to the molecular function.

screening of barley databases has identified two SBP genes targeted by miR156 for which two nucleotide variations occur in critical positions (11-12). If these SNPs will be experimentally confirmed, they could have the effect of destabilizing the interaction between the miRNA and the mRNA, which could consequently avoids cleavage and lead to phenotypical variations in developmental features or in the resistance to viral infection.

A SNP also occurs in a crucial point of the experimentally confirmed NAC1 target for miR164. NAC1 is a transcription factor involved in shoot apical meristem formation and auxin-mediated lateral root formation. Guo et al. showed that the overexpression of miR164 leads to reduced lateral rooting; conversely the disruption of the regulation mediated by this miRNA increases the number of lateral roots [28]. The authors have reported that miR164 directs cleavage in vivo at a position complementary to the $10^{\text {th }}$ nucleotide from the $5^{\prime}$ end of the mature sequence [28]. The SNP found in barley is in the $11^{\text {th }}$ position, therefore it is likely to prevent the cleavage and produce phenotypic effects on root development.

SNPs have been identified also in other two conserved miRNA targets, TIR1 and AGO4, targeted respectively by miR393 and miR408. TIR1 is an auxin-receptor negatively regulated by miRNAs in response to bacterial flagellin, as a defence mechanism against Pseudomonas syringae [29]. AGO4 is a protein involved in the siRNA mediated gene silencing, and it is required for the resistance to the same pathogen [30]. Therefore, miR393 and miR408 are likely to work in a coupled manner during P. syringae infection. The two SNPs identified are in the $12^{\text {th }}$ position and could potentially alter the levels of pathogens resistance. 
Table 5 Unigene clusters candidate to encode for miRNAs

\begin{tabular}{|c|c|c|c|c|c|c|c|c|c|c|}
\hline \multirow[b]{2}{*}{ Unigene cluster } & \multicolumn{6}{|c|}{ Features of the precursors identified } & \multicolumn{4}{|c|}{ Best scoring alignment with miRBase precursors } \\
\hline & $\Delta \mathrm{G}(\mathrm{kcal} / \mathrm{mol})$ & MFEI & NM & ML & $P L$ & Arm & Accession number & miRNA & Score & e-value \\
\hline Hv.1306 & -72.8 & 0.89 & 2 & 21 & 167 & $3^{\prime}$ & MI0006178 & tae-MIR444 & 736 & $1 \times 10^{-54}$ \\
\hline Hv.5064 & -22.0 & 1.00 & 2 & 18 & 68 & $3^{\prime}$ & MI0006199 & tae-MIR1137 & 168 & $4 \times 10^{-8}$ \\
\hline Hv.7117 & -74.9 & 0.96 & 3 & 21 & 115 & $5^{\prime}$ & MI0011565 & bdi-MIR397 & 196 & $5 \times 10^{-10}$ \\
\hline Hv.8158 & -60.7 & 0.88 & 3 & 21 & 92 & $5^{\prime}$ & MI0001763 & sof-MIR168a & 393 & $1 \times 10^{-26}$ \\
\hline Hv.14657 & -31.5 & 1.85 & 2 & 21 & 69 & $3^{\prime}$ & MI0006183 & tae-MIR1121 & 241 & $3 \times 10^{-14}$ \\
\hline Hv.15131 & -51.1 & 0.90 & 2 & 21 & 129 & $3^{\prime}$ & MI0006976 & osa-MIR444d & 431 & $2 \times 10^{-29}$ \\
\hline Hv.16635 & -91.7 & 0.92 & 3 & 21 & 200 & $3^{\prime}$ & MI0006170 & tae-MIR159a & 805 & $2 \times 10^{-60}$ \\
\hline Hv.22601 & -34.0 & 0.92 & 4 & 22 & 97 & $3^{\prime}$ & Ml0006192 & tae-MIR1130 & 179 & $9 \times 10^{-9}$ \\
\hline Hv.28058 & -63.8 & 1.60 & 2 & 24 & 129 & $3^{\prime}$ & MI0006182 & tae-MIR1120 & 147 & $7 \times 10^{-6}$ \\
\hline Hv.29065 & -53.6 & 1.07 & 4 & 22 & 131 & $5^{\prime}$ & MI0006199 & tae-MIR1137 & 182 & $8 \times 10^{-9}$ \\
\hline Hv.29519 & -42.9 & 1.02 & 2 & 21 & 96 & $3^{\prime}$ & MI0006192 & tae-MIR1130 & 144 & $8 \times 10^{-6}$ \\
\hline Hv.30469 & -39.0 & 0.91 & 3 & 21 & 117 & $5^{\prime}$ & Ml0006199 & tae-MIR1137 & 141 & $2 \times 10^{-5}$ \\
\hline
\end{tabular}

For each cluster, the table shows details about the putative precursors: the free energy $\Delta \mathrm{G}$, the minimal folding free energy index (MFEl), the number of mismatches in miRNA/miRNA* duplex (NM), the mature length ( $\mathrm{ML})$, the precursor length $(\mathrm{PL})$ and the location of mature miRNA $\left(3^{\prime}\right.$ or $\left.5^{\prime}\right)$. Moreover, it is also reported the more similar known precursor in miRBase, with the alignment score and $\mathrm{p}$-value.

SNPs were also found in previously not reported miRNA targets, such as the AWPM-19-like protein matching to the miRNA 1134. AWPM-19 accumulates in wheat plasma membrane during cold acclimation in response to abscisic acid [31]. If this miRNA really controls the synthesis of this protein, a deleterious SNP in the $11^{\text {th }}$ position could then change resistance to cold stress.

\section{Conclusions}

This study has thus provided an update of the information on barley miRNAs and their targets representing a foundation for future studies.

Novel putative target genes have been identified and most of them are involved in stress and hormone response. Indeed, the role of plant miRNAs in abiotic and biotic stress response as well as in auxin signalling is well known [32,33]. In particular, protein kinases such as protein kinase $\mathrm{C}$ and serine/threonine kinase, known to be important regulator on abiotic stress resistance, are largely present in novel microRNA/target pairs identified.

The results have also shown that microRNA target sites can be an interesting source for the identification of functional genetic variability, representing an interesting source of candidate molecular markers for application in barley breeding. Putative polymorphisms have now to be verified by amplification and sequencing of the target sequences on a larger set of genotypes.

Sequence analysis based on known miRNAs can obviously give insights only on conserved mRNAs and related targets. Future work will thus be based on the construction of a degradome library for parallel analysis of RNA end (PARE), a powerful approach for high-throughput identification/validation of conserved and non conserved targets.

\section{Methods}

miRNA reference dataset

The initial miRNA dataset has been obtained by extracting the mature sequences (1929 entries) of the Viridiplantae group from the miRBase release $13 \mathrm{http} / / /$ www.mirbase.org[34]. By removing identical mature sequences, the size of this dataset has been subsequently reduced to 1014 non-redundant sequences related to 468 miRNA families.

\section{Searching for mature miRNAs matching sequences in barley}

The full collection of non-redundant mature miRNA sequences was used in a BLASTn search against dbEST http://www.ncbi.nlm.nih.gov, accepting a number of mismatch lower than 4.

The set of miRNA mature sequences (including the identical sequences removed at the first step of the work) with at least one matching EST have been classified on the basis of the species of origin. The binomial distribution was used to assess the statistical significance for the represented plant species; this allowed identifying those species chosen from the initial dataset more or less frequently than random. Four different thresholds for the $\mathrm{p}$-values were applied $(0.05,0.01$, $0.005,0.001$ ).

Matching ESTs have then been related to Unigene clusters and the corresponding annotations were recorded (if available). The GO slimmer tool available on the Gene 
Table 6 Putative polymorphisms identified at miRNA target sites and inside miRNA mature sequences

\begin{tabular}{|c|c|c|c|c|c|}
\hline $\begin{array}{l}\text { miRNA } \\
\text { family }\end{array}$ & $\begin{array}{l}\text { miRNA } \\
\text { name }\end{array}$ & Unigene & Unigene cluster annotation & $\begin{array}{l}\text { Putative Polymorphisms } \\
\text { at miRNA target site }\left(5^{\prime}-3^{\prime}\right)\end{array}$ & $\begin{array}{l}\text { Barley miRNA mature sequence } \\
\qquad\left(5^{\prime}-3^{\prime}\right)\end{array}$ \\
\hline \multirow[t]{2}{*}{156} & miR156 & Hv. 5875 & protein coding (SBP domain) & $\begin{array}{l}\text { \#GTGCTCTCT(C) } \\
\text { CTCTTCTGTCA }\end{array}$ & UGACAGAAGAGAGAGAGCAC (12) \\
\hline & miR156 & Hv.21387 & $\begin{array}{l}\text { SPL2 (SQUAMOSA PROMOTER BINDING } \\
\text { PROTEIN-LIKE 2) }\end{array}$ & $\begin{array}{c}\text { \#ATGCTCTC(G)TC(T)TC(G)} \\
\text { TTCTGTCA }\end{array}$ & $\begin{array}{l}\text { UGACAGAAGAGAGAGAGCAU } \\
\qquad(9-11-13)\end{array}$ \\
\hline 164 & miR164 & Hv.28795 & NAC domain containing protein & $\begin{array}{l}\text { \#AGCAAGTGCCC(A) } \\
\text { TGCTTCTCCA }\end{array}$ & UGGAGAAGCAGGGCACUUGCU (11) \\
\hline \multirow[t]{2}{*}{169} & $\operatorname{miR} 169$ & Hv.13681 & $\begin{array}{l}\text { CCAAT-binding transcription factor (CBF-B/NF- } \\
\text { YA) family protein }\end{array}$ & $\begin{array}{l}\text { \#CAGGCAACTCATCCTTGGCT } \\
\text { (C)T }\end{array}$ & AAGCCAAGGAUGAGUUGCCUG (2) \\
\hline & $\operatorname{miR} 169$ & Hv.9532 & $\begin{array}{l}\text { CCAAT-binding transcription factor (CBF-B/NF- } \\
\text { YA) family protein }\end{array}$ & $\begin{array}{c}\text { \#GGCAATTCATCCTTGGC(T) } \\
\pi\end{array}$ & AAGCCAAGGAUGAAUUGCC (3) \\
\hline 393 & miR393 & Hv.2498 & $\begin{array}{c}\text { TIR1 (TRANSPORT INHIBITOR RESPONSE 1), } \\
\text { ubiquitin-protein ligase }\end{array}$ & $\begin{array}{c}\text { \#G(C)ACAATGCG(T)ATCCC } \\
(+C T) T T G G A\end{array}$ & $\begin{array}{l}\text { UCCAAA()GGGAUCGCAUUGUC } \\
(6-12-20)\end{array}$ \\
\hline 396 & miR396 & Hv.9742 & WRC, QLQ & $\begin{array}{c}\text { \#GTTCAAG(A) } \\
\text { AAAGCCTGTGGA }\end{array}$ & UCCACAGGCUUUCUUGAAC(13) \\
\hline \multirow[t]{2}{*}{408} & miR408 & Hv.20945 & ARGONAUTE like superfamily & $\begin{array}{l}\text { \#CAGGGCAG(T) } \\
\text { AGGCAGTGCAG }\end{array}$ & CUGCACUGCCUCUGCCCUG (12) \\
\hline & miR408 & $\begin{array}{c}\text { AutoSNP } \\
\text { contig } \\
2094\end{array}$ & Plastocyanin & $\begin{array}{l}\text { \#CAGGGAAGAGGCA(C) } \\
\text { GTGCGG }\end{array}$ & CCGCACU(G)GCCUCUUCCCUG (7) \\
\hline 444 & miR444 & Hv.16297 & / & & $\begin{array}{c}{ }^{*} \text { GCAGUUGCU(C)GCCUCAAGCUU } \\
\text { (9) }\end{array}$ \\
\hline \multirow[t]{4}{*}{818} & miR818 & Hv.11323 & protein coding & $\begin{array}{l}\text { \#CCGTCCCATAA }(C C) \\
\text { TATAAGGG }\end{array}$ & CCCUUAUAUUAUGGGACGG (9) \\
\hline & $\operatorname{miR} 1436$ & Hv.8351 & protein coding & $\begin{array}{l}\text { \#ACTCCCTCC(T) } \\
\text { GTCCCATAAT }\end{array}$ & AUUAUGGGACGGAGGGAGU (11) \\
\hline & miR1436 & Hv.11323 & protein coding & $\begin{array}{c}\text { \#ACTCCCTCCGTCCCATAA- } \\
\text { (CC)T }\end{array}$ & A-UUAUGGGACGGAGGGAGU (2) \\
\hline & miR1439 & Hv.23816 & exo-endo-phos superfamily & $\begin{array}{c}\# \\
\text { AATACTCACTCCGTCCCAAAA }\end{array}$ & UUUUGGGACGGAGUGAGUAUU (1) \\
\hline
\end{tabular}

$(\mathrm{G})$

\begin{tabular}{|c|c|c|c|c|c|}
\hline & miR1439 & Hv.11224 & tatD-related deoxyribonuclease family protein & $\begin{array}{c}\text { \#TACTCACTCCGTTCCA(T) } \\
\text { AAA }\end{array}$ & UUUUGGAACGGAGUGAGUA (4) \\
\hline 821 & miR821 & Hv.3660 & GDH1 (Glutamate dehydrogenase) & \#TCAA(C)CAAAAAAGTTGAAT & AUUCAACUUUUUUGUUGA (15) \\
\hline 1030 & miR1030 & Hv.14867 & RNA recognition motif (RRM)-containing protein & $\begin{array}{c}\text { \#TGGT(G) } \\
\text { GCAGGTGCAGGTGCAGG }\end{array}$ & CCUGCACCUGCACCUGCACCA (18) \\
\hline \multirow[t]{5}{*}{1119} & $\operatorname{miR} 1119$ & Hv.29226 & / & & $\begin{array}{c}\text { *UGGC(-)A(C) } \\
\text { CGGCGCGAUGCUCAGUCA(-)G(C) } \\
(4-5-23-24)\end{array}$ \\
\hline & miR1119 & Hv.29225 & protein coding & $\begin{array}{c}\text { \#CTGAC(A) } \\
\text { TGAGCATCGCGCCGTGCCA }\end{array}$ & $\begin{array}{c}\text { UGGCACGGCGCGAUGCUCAGUCAG } \\
(20)\end{array}$ \\
\hline & miR1119 & Hv.27666 & protein coding & $\begin{array}{c}\# \mathbf{C}(\mathrm{T}) \mathrm{TGAC}(\mathbf{T} / \mathrm{A}) \mathrm{TG}(\mathrm{A}) \mathbf{A}(\mathrm{G}) \\
\mathrm{GCA}(\mathrm{T}) \mathrm{TCGCGCCGTGCC}\end{array}$ & $\begin{array}{c}\text { GGCACGGCGCGAUGCUCAGUCAG } \\
(13-16-17-19-23) \\
\end{array}$ \\
\hline & miR1119 & Hv.23343 & $\begin{array}{c}\text { molybdenum cofactor sulfurase family protein, } \\
\text { superfamily }\end{array}$ & $\begin{array}{l}\text { \#CT(G)G(T)A(G)CT(C) } \\
\text { GAGCATCGCGCCGTGCC }\end{array}$ & $\begin{array}{c}\text { GGCACGGCGCGAUGCUCAGUCAG } \\
(18-20-21-22) \\
\end{array}$ \\
\hline & miR1119 & Hv.29210 & protein coding & $\begin{array}{c}\# \mathbf{T}(\mathrm{G}) \mathrm{GGCACG}(\mathrm{A}) \mathrm{GC}(\mathrm{T}) \\
\mathrm{GCGAT}(\mathrm{A}) \mathrm{GCTCAG}(\mathrm{A}) \mathrm{TCA} \mathbf{G} \\
(\mathrm{A})\end{array}$ & $\begin{array}{c}\text { CUGACUGAGCAUCGCGCCGUGCCA } \\
(1-5-11-16-18-24)\end{array}$ \\
\hline \multirow[t]{4}{*}{1120} & $\operatorname{miR} 1121$ & Hv.464 & serine/threonine kinase & $\begin{array}{c}\# \mathbf{A}(\mathrm{G}) \mathbf{A}(\mathrm{G}) \\
\text { GAGCGTTIAGATCACTA }\end{array}$ & UAGUGAUCUAAACGCUCUU (18-19) \\
\hline & miR1121 & Hv.6581 & protein coding & $\begin{array}{l}\text { \#TAAGAGCGTTAAGATCACT } \\
\text { (C)A }\end{array}$ & UAGUGAUCUAAACGCUCUUA (2) \\
\hline & miR1121 & Hv.6532 & $\begin{array}{l}\text { ATPase-Plipid, haloacid dehalogenase-like } \\
\text { hydrolase family protein }\end{array}$ & $\begin{array}{c}\text { \#TAAG (A) } \\
\text { AGTGTITAGATCACTACT }\end{array}$ & $\begin{array}{l}\text { AGUAGUGAUCUAAACACUCUUA } \\
\text { (19) }\end{array}$ \\
\hline & miR1121 & Hv.5064 & / & & ${ }^{*}$ UAGUACAAAGUUG(C)AGUCA (13) \\
\hline
\end{tabular}


Table 6 Putative polymorphisms identified at miRNA target sites and inside miRNA mature sequences (Continued)

\begin{tabular}{|c|c|c|c|c|c|}
\hline 1122 & $\operatorname{miR} 1128$ & Hv.14876 & ARF-GAP DOMAIN, C2 superfamily & $\begin{array}{c}\# T T \mathbf{G}(\mathrm{T}) \mathrm{GG}(\mathrm{A}) \mathrm{ACGGA}(\mathrm{G}) \\
\mathrm{GGGAGTAGTA}\end{array}$ & $\begin{array}{l}\text { UACUACUCCCUCCGUCCCAAA (11- } \\
\text { 16-18) }\end{array}$ \\
\hline & miR1133 & Hv.12091 & $\begin{array}{l}\text { oxidoreductase, zinc-binding dehydrogenase } \\
\text { family protein }\end{array}$ & $\begin{array}{c}\text { \#TTTGGG(A) } \\
\text { ACGGAGGGAGTAC(-)TAT }\end{array}$ & $\begin{array}{l}\text { AUAGUACUCCCUCCGUCCCAAA (3- } \\
16)\end{array}$ \\
\hline & miR1133 & Hv.28555 & $\begin{array}{c}\text { serine/threonine protein kinase, PKc-like } \\
\text { superfamily }\end{array}$ & $\begin{array}{c}\text { \#TTTCGGACAGAGGG(T) } \\
\text { AGTATAT }\end{array}$ & AUAUACUCCCUCUGUCCGAAA (8) \\
\hline & miR1135 & Hv. 18515 & ubiquitin family protein & $\begin{array}{c}\text { \#TTC(G) } \\
\text { GGAATTACTTGTCGCA }\end{array}$ & UGCGACAAGUAAUUCCGAA (17) \\
\hline \multirow[t]{6}{*}{1134} & miR1134 & Hv.22973 & $\begin{array}{c}\text { Octopine synthase binding factor1, ATBZIP53 } \\
\text { (BASIC REGION/LEUCINE ZIPPER MOTIF 53), DNA } \\
\text { binding/protein heterodimerization/sequence- } \\
\text { specific DNA binding/transcription factor }\end{array}$ & $\begin{array}{c}\text { \#TCTTCTTCTTCTTCTTG(C) } \\
\text { TTC(-)TTG }\end{array}$ & $\begin{array}{c}\text { CAAGAACAAGAAGAAGAAGAAGA } \\
(4-5-6-7)\end{array}$ \\
\hline & $\operatorname{miR} 1134$ & Hv.9579 & $\begin{array}{l}\text { L-asparaginase, putative/L-asparagine } \\
\text { amidohydrolase, putative }\end{array}$ & $\begin{array}{c}\text { \#TC(G)T(C) } \\
\text { TCTTCTTCTTGGTGTTGGTG }\end{array}$ & $\begin{array}{c}\text { CACCAACACCAAGAAGAAGAAGA } \\
(21-22)\end{array}$ \\
\hline & miR1134 & Hv.26138 & AWPM-19-like membrane family protein & $\begin{array}{l}\text { \#TCTTCTTC(G)TTCTT(A) } \\
\text { GTCGTTGTTG }\end{array}$ & $\begin{array}{c}\text { CAACAACGACAAGAAGAAGAAGA } \\
(11-16)\end{array}$ \\
\hline & miR1134 & Hv.24001 & dehydrin family protein & $\begin{array}{c}\# T T C T T C T T C T T G T T G T T T T T(-) \\
\text { G }\end{array}$ & CAAAAACAACAAGAAGAAGAA (2) \\
\hline & $\operatorname{miR} 1134$ & Hv.8025 & / & & $\begin{array}{c}* \text { *UCUUCUUCUUUUGUUGUUGU(C) } \\
\text { UG (20) }\end{array}$ \\
\hline & $\operatorname{miR} 1134$ & Hv.5763 & / & & $\begin{array}{c}{ }^{*} \text { CUUC(G) } \\
\text { UUCCUCUUGUUGUUGUUG (4) }\end{array}$ \\
\hline 1871 & miR1871 & Hv.28885 & protein coding & $\begin{array}{c}\text { \# C(T) } \\
\text { AACATGATATCAGAGCCA }\end{array}$ & UGGCUCUGAUAUCAUGUUG (19) \\
\hline
\end{tabular}

Letters in bold refer to SNPs represented by at least two independent sequences, while the numbers in brackets refer to the position of the SNP in the sequence. Plus means insertion, minus means deletion.
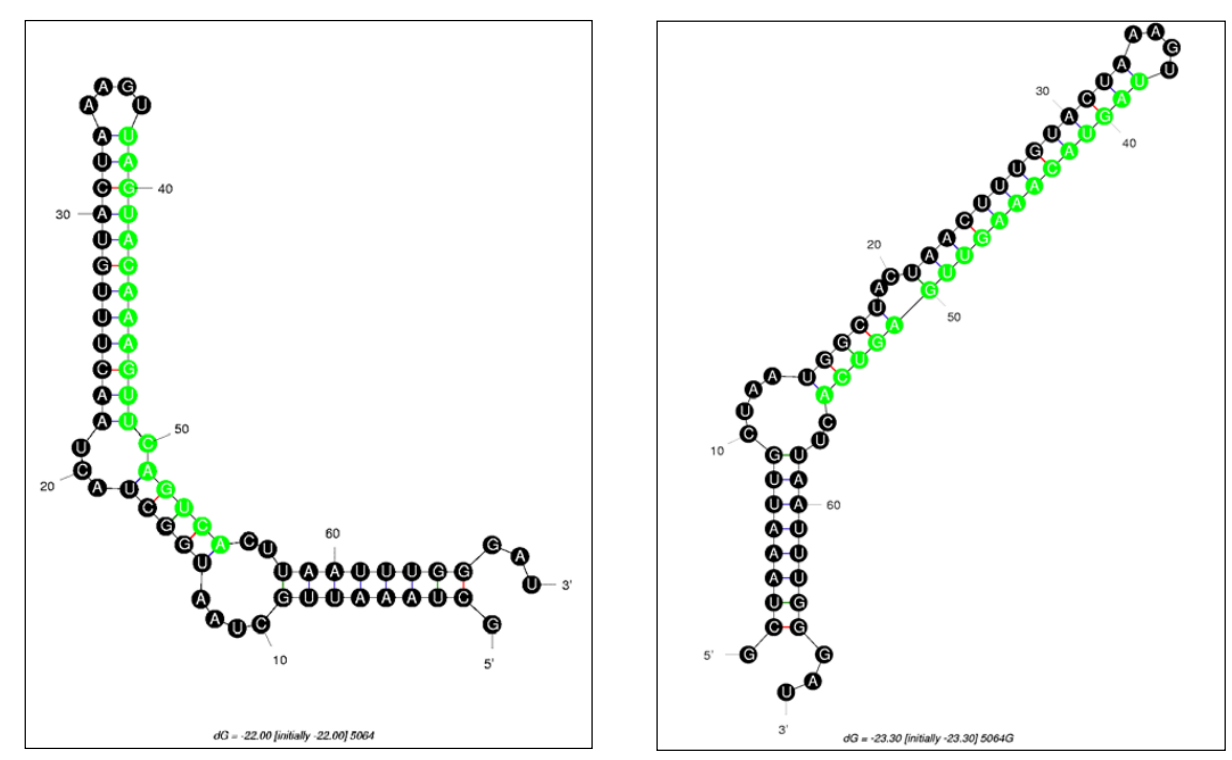

$\begin{array}{ccccccc}\Delta \mathrm{G} & \begin{array}{c}\text { length } \\ (\mathrm{nt})\end{array} & \% \mathrm{GC} & \text { MFEI } & \begin{array}{c}\text { mismatch } \\ \text { miRNA/miRNA* }\end{array} & \begin{array}{c}\text { arm of the } \\ \text { mature } \\ \text { sequence }\end{array} \\ \operatorname{miR1137}(\mathbf{k}) & -22.0 & 68 & 32.35 & 1.00 & 2 & 3^{\prime} \\ \operatorname{miR1137}(G) & -23.3 & 68 & 32.35 & 1.06 & 2 & 3^{\prime}\end{array}$

Figure 3 Predicted secondary structures of the two variants of the miR1137 precursor identified in the Unigene cluster Hv.5064. The variants with a $C$ and a $G$ in the $13^{\text {th }}$ position are respectively reported in the left and in the right side of the figure. The table shows for each variant: the free energy $\Delta G$, the length of the precursor, the GC content, the MFEl (Minimal Folding Energy Index), the number of mismatches between the mature sequence and the paired miRNA* passenger and the arm of the hairpin where the mature sequence is located. 


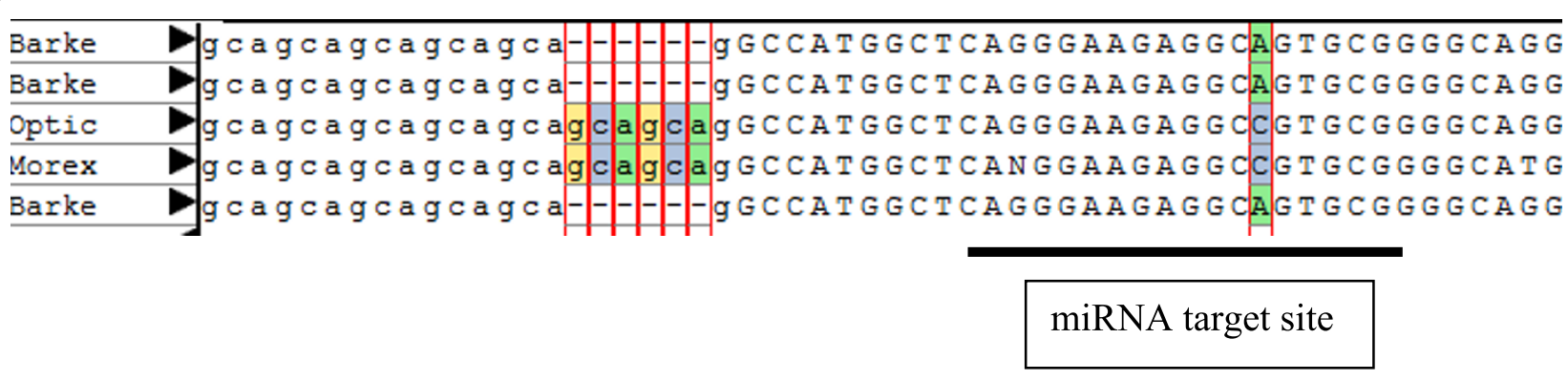

Figure 4 SNP identified in contig2094 within the target site for miRNA 408. In this multiple alignment performed with AutoSNP, two cultivars (Optic and Morex) report the same allelic variant as part of a haplotype including a SSR polymorphism located upstream the target sequence.

Ontology website http://www.geneontology.org has been used to identify the GO slim terms more represented in the set of potential targets on the basis of the Unigene cluster annotations. For this analysis the Plant GO Slim subset has been used.

\section{Identification of putative miRNA precursors}

True miRNA precursors should have both a mature sequence on one arm of the hairpin and a paired passenger sequence (called miRNA*) on the opposite arm. To assess these features the precursor sequences were extracted from the consensus sequences, obtained by the Sequencer Software (Gene Codes) on Unigene cluster assemblies, by cutting 13 nt before the $5^{\prime}$ hit and 13 nt after the 3' hit, since this region (called the pri-extension region of the hairpin) was recently shown to have this average length in plants [35]. In order to predict the secondary structure of the precursors, the software mfold 3.2, free available at http://mfold.bioinfo.rpi.edu/ cgi-bin/rna-form1.cgi, was used [36]. The minimal folding free energy index (MFEI) and the GC content were calculated for each sequence.

All the sequences with a MFEI greater than 0.85 were considered potential miRNA precursors [37]; besides, only 4 mismatches were allowed between the mature sequence and the passenger sequence, and only few and small asymmetric bulges were accepted [38].

\section{Identification of SNPs/indels at miRNA target sites}

Polymorphisms in target genes have been searched through a comparison of the ESTs belonging to the same Unigene cluster. Each cluster has been assembled by Sequencer Software (Gene Codes) and polymorphisms have been searched on miRNA complementary sequence sites.

AutoSNP database http://autosnpdb.qfab.org.au was also screened using target gene annotations as contigsearching keywords.

\section{Additional material}

Additional file 1: BLAST results. Alignments identified between plant miRNAs and barley ESTs.

Additional file 2: Barley mature miRNA sequences. Aligned barley mature miRNA sequences grouped on the basis of the miRNA family. Families for which only one barley EST has been found to match are not reported in this file.

Additional file 3: microRNA matching ESTs. List of ESTs matching the mature miRNA sequences. ESTs that present more than 1 target site for the same miRNA family or for different miRNA families have been highlighted.

Additional file 4: Secondary structures of the putative miRNA precursors. Predicted structures of the identified putative miRNA precursors. The prediction was performed with mfold.

\section{Acknowledgements}

This work has been supported by "MIRNA" project and "Mappa 5A" project (Italian Ministry for Agriculture). The authors thank Mr. Renzo Alberici and Miss Alice Martini for the technical assistance provided.

\section{Authors' contributions}

MC identified barley microRNAs and targets and highlighted the putative novel ones. He was also involved in polymorphism searching. PF conceived, designed and coordinated the work and wrote the manuscript. AS gave a contribution for polymorphisms searching and alignment of barley mature microRNA sequences. PB and AL prepared the additional material and gave a contribution to bioinformatics analysis. LC contributed in the design and discussion of the work and assisted in drafting the manuscript. All authors read and approved the final manuscript.

Received: 30 April 2010 Accepted: 22 October 2010 Published: 22 October 2010

\section{References}

1. Bartel DP: MicroRNAs: genomics, biogenesis, mechanism and function. Cell 2004, 116:281-297.

2. Dryanova A, Zakharov A, Gulick PJ: Data mining for miRNAs and their targets in the Triticeae. Genome 2008, 51:433-443.

3. Sunkar R, Jagadeeswaran $\mathrm{G}$ : In silico identification of conserved microRNAs in large number of diverse plant species. BMC Plant Biol 2008, 8:37.

4. Schreiber AW, Sutton T, Caldo RA, Kalashyan E, Lovell B, Mayo G, Muehlbauer GJ, Druka A, Waugh R, Wise RP, Langridge P, Baumann U: Comparative transcriptomics in the Triticeae. BMC Genomics 2009, 10:285. 
5. Zhang BH, Pan XP, Wang QL, Cobb GP, Anderson TA: Identification and characterization of new plant microRNAs using EST analysis. Cell Res 2005, 15(5):336-360.

6. German MA, Pillay M, Jeong D, Hetawal A, Luo S, Janardhanan P, Kannan $\mathrm{V}$, Rymarquis LA, Nobuta K, German R, De Paoli E, Lu C, Schroth G, Meyers BC, Green PJ: Global identification of microRNA-target RNA pairs by parallel analysis of RNA ends. Nat Biotechnol 2008, 26(8):941-946.

7. Zeng C, Wang W, Zheng Y, Chen X, Bo W, Song S, Zhang W, Peng M: Conservation and divergence of microRNAs and their functions in Euphorbiaceous plants. Nucleic Acids Res 2009, 38(3):981-995.

8. Xie K, Wu C, Xiong L: Genomic organization, differential expression and interaction of SQUAMOSA promoter-binding-like transcription factors and microRNA156 in rice. Plant Physiol 2006, 142:280-293.

9. Picoult-Newberg L, Ideker TE, Pohl MG, Taylor SL, Donaldson MA, Nickerson DA, Boyce-Jacino M: Mining SNPs from EST databases. Genome Res 1999, 9:167-174.

10. Zhang B, Pan X, Cannon CH, Cobb GP, Anderson TA: Conservation and divergence of plant microRNA genes. Plant J 2006, 46:243-259.

11. Yao Y, Guo G, Ni Z, Sunkar R, Du J, Zhu JK, Sun Q: Cloning and characterization of microRNAs from wheat (Triticum aestivum L.). Genome Biol 2007, 8:R96.

12. Zhou M, Gu L, Li P, Song X, Wei L, Chen Z, Cao X: Degradome sequencing reveals endogenous small RNA targets in rice (Oryza sativa L., ssp. Indica). Front Biol 2010, 5(1):67-90.

13. Grun D, Wang YL, Langenberger D, Gunsalus KC, Rajewsky N: microRNA target predictions across seven Drosophila species and comparison to mammalian targets. PLoS Comput Biol 2005, 1:e13.

14. Zhang B, Pan X, Anderson TA: Identification of 188 conserved maize microRNAs and their targets. FEBS Lett 2006, 580:3753-3762.

15. Jones-Rhoades MW, Bartel DP, Bartel B: MicroRNAs and their regulatory roles in plants. Annu Rev Plant Biol 2006, 57:19-53.

16. Guo Q, Xiang A, Yang Q, Yang Z: Bioinformatic identification of microRNAs and their target genes from Solanum tuberosum expressed sequence tags. Chinese Sci Bull 2007, 52(17):2380-2389.

17. Xie FL, Huang SQ, Guo K, Xiang AL, Zhu YY, Nie L, Yang ZM: Computational identification of novel microRNAs and targets in Brassica napus. FEBS Lett 2007, 581:1464-1474.

18. Nair SK, Wang N, Turuspekov $Y$, Pourkheirandish $M$, Sinsuwongwat $S$, Chen G, Sameri M, Tagiri A, Honda I, Watanabe Y, Kanamori H, Wicker T, Stein N, Nagamura Y, Matsumoto T, Komatsuda T: Cleistogamous flowering in barley arises from the suppression of microRNA-guided HvAP2 mRNA cleavage. PNAS 2010, 107:480-496.

19. Ehrenreich IM, Purugganan MD: Sequence variation of microRNAs and their binding sites in Arabidopsis. Plant Physiol 2008, 146:1974-1982.

20. Guo X, Gui Y, Wang Y, Zhu Q, Helliwell C, Fan L: Selection and mutation on microRNA target sequences during rice evolution. BMC Genomics 2008, 9:454.

21. Saunders MA, Liang H, Li WH: Human polymorphisms at microRNAs and microRNA target sites. PNAS 2007, 104:3300-3305.

22. Sethupathy P, Collins FS: MicroRNA target site polymorphisms and human disease. Trends Genet 2008, 24:489-497.

23. Gardner PP, Vinther J: Mutation of miRNA target sequences during human evolution. Trends Genet 2008, 24:262-265.

24. Rhoades MW, Reinhart BJ, Lim LP, Burge CB, Bartel B, Bartel DP: Prediction of plant microRNA targets. Cell 2002, 110:513-520

25. Batley J, Barker G, O'Sullivan H, Edwards K, Edwards D: Mining for single nucleotide polymorphisms and insertions/deletions in maize expressed sequence tag data. Plant Physiol 2003, 132:84-91.

26. Kasschau KD, Xie Z, Allen E, Llave C, Chapman EJ, Krizan KA, Carrington JC P1/HC-Pro, a viral suppressor of RNA silencing, interferes with Arabidposis development and miRNA function. Dev Cell 2003, 4:205-217.

27. Chen J, Li WX, Xie D, Peng JR, Ding SW: Viral virulence protein suppresses RNA silencing-mediated defense but upregulates the role of microRNA in host gene expression. Plant Cell 2004, 16:1302-1313.

28. Guo HS, Xie Q, Fei JF, Chua NH: MicroRNA directs mRNA cleavage of the transcription factor NAC1 to downregulate auxin signals for Arabidopsis lateral root development. Plant Cell 2005, 17:1376-1386.

29. Navarro L, Dunoyer P, Jay F, Arnold B, Dharmasiri N, Estelle M, Voinnet O, Jones JDG: A plant miRNA contributes to antibacterial resistance by repressing auxin signalling. Science 2006, 312:436-439.
30. Agorio A, Vera P: ARGONAUTE4 is required for resistance to Pseudomonas syringae in Arabidopsis. Plant Cell 2007, 19:3778-3790.

31. Koike M, Takezawa D, Arakawa K, Yoshida S: Accumulation of 19-kDa plasma membrane polypeptide during induction of freezing tolerance in wheat suspension-cultured cells by abscisic acid. Plant Cell Physiol 1997, 38:707-716.

32. Martin RC, Liu PP, Goloviznina NA, Nonogaki H: microRNA, seeds, and Darwin?: diverse function of microRNA in seed biology and plant response to stress. J Exp Bot 2010, 69:2229-2234.

33. Teotia PS, Mukherjee SK, Mishra NS: Fine tuning of auxin signalling by miRNAs. Physiol Mol Biol Plants 2008, 14:81-90.

34. Griffiths-Jones S, Saini HK, van Dongen S, Enright AJ: miRBase: tools for microRNA genomics. NAR 2008, 36 Database: D154-D158.

35. Kadri S, Hinman V, Benos PV: HHMMiR: efficient de novo prediction of microRNAs using hierarchical hidden Markov models. Bioinformatics 2009, 10(Suppl I):S35.

36. Zuker M: mfold web server for nucleic acid folding and hybridization prediction. Nucleic Acids Res 2003, 31(13):3406-15.

37. Zhang BH, Pan XP, Cox SB, Cobb GP, Anderson TA: Evidence that miRNAs are different from other RNAs. Cell Mol Life Sci 2006, 63:246-254.

38. Meyers BC, Axtell MJ, Bartel B, Bartel DP, Baulcombe D, Bowman JL, Cao X, Carrington JC, Chen X, Green PJ, Griffiths-Jones S, Jacobsen SE, Mallory AC, Martienssen RA, Poethig RS, Qi Y, Vaucheret $H$, Vonnet O, Watanabe $Y$, Weigel D, Zhu JK: Criteria for annotation of plant microRNAs. Plant Cell 2008, 20:3186-3190.

39. Jones-Rhoades MW, Bartel DP: Computational identification of plant microRNAs and their targets, including a stress-induced miRNA. Molecular Cell 2004, 14:787-799.

40. Puzey JR, Kramer EM: Identification of conserved Aquilegia coerulea microRNAs and their targets. Gene 2009, 448(1):46-56.

41. Unver T, Budak $\mathrm{H}$ : Conserved microRNAs and their targets in model grass species Brachypodium distachyon. Planta 2009, 230(4):659-69.

42. Park W, Li J, Song R, Messing J, Chen X: CARPEL FACTORY, a Dicer homolog, and HEN1, a novel protein, act in microRNA metabolism in Arabidopsis thaliana. Current Biology 2002, 12(17):1484-1495.

43. Trindade I, Capitão C, Dalmay T, Fevereiro MP, Dos Santos DM: miR398 and miR408 are up-regulated in response to water deficit in Medicago truncatula. Planta 2010, 231(3):705-716.

44. Lu S, Sun YH, Shi R, Clark C, Li LG, Chiang VL: Novel and mechanical stress-responsive microRNAs in Populus trichocarpa that are absent from Arabidopsis. Plant Cell 2005, 17:2186-2203.

45. Li YF, Zheng Y, Addo-Quaye C, Zhang L, Saini A, Jagadeeswaran G, Axtell MJ, Zhang WX, Sunkar R: Transcriptome-wide identification of microRNA targets in rice. Plant J 2010, 62(5):742-759.

46. Zhang LF, Chia JM, Kumari S, Stein JC, Liu ZJ, Narechania A, Maher CA, Guill K, McMullen MD, Ware D: A genome-wide characterization of microRNA genes in maize. PLoS Genet 2009, 5(11):e1000716.

47. Rajagopalan R, Vaucheret H, Trejo J, Bartel DP: A diverse and evolutionarily fluid set of microRNAs in Arabidopsis thaliana. Genes and Development 2006, 20(24):3407-3425

48. Lacombe S, Nagasaki H, Santi C, Duval D, Piégu B, Bangratz M, Breitler JC, Guiderdoni E, Brugidou C, Hirsch J, Cao XF, Brice C, Panaud O, Karlowski WM, Sato Y, Echeverria M: Identification of precursor transcripts for 6 novel miRNAs expands the diversity on the genomic organisation and expression of miRNA genes in rice. BMC Plant Biol 2008, 8:123.

doi:10.1186/1471-2164-11-595

Cite this article as: Colaiacovo et al: A computational-based update on microRNAs and their targets in barley (Hordeum vulgare L.). BMC Genomics 2010 11:595. 